Sturzprävention

\section{Intelligente Haltungserkennung am Rollator}

— Das Bundesministerium für Bildung und Forschung (BMBF) fördert aktuell ein Forschungsprojekt, mit dem ein intelligentes Unterstützungssystem zur Sturzprävention bei der Benutzung von Rollatoren entwickelt werden soll, mit 912.000€. Vier Partner aus Wissenschaft, Gesundheit und Industrie setzen das Projekt um. Koordinator ist Budelmann Elektronik in Münster, außerdem arbeiten der Bremer DFKI-Forschungsbereich Cyber-Physical Systems, der Gesundheit Nord Klinikverbund Bremen sowie die diesem Projekt. Das Vorhaben mit dem Namen ModESt („Rollator-Modul zur HaltungsErkennung und Sturz-Prävention“) läuft über drei Jahre. Dabei soll ein Unterstützungssystem sowohl für ältere Menschen mit Haltungsproblemen als auch für kranke Menschen entwickelt werden, das durch eine intelligente Sturzprävention dazu beiträgt, Mobilitätseinschränkungen und Unsicherheiten zu verhindern. TOPRO GmbH aus Fürstenfeldbruck an
Ziel ist es, eine Elektronikbox zu entwickeln, die am Rollator befestigt wird. Diese soll die Haltung des Nutzers analysieren, bewerten und anschließend bei der Korrektur helfen. Zunächst überprüft die Analysefunktion die Positionierung des Nutzers in Relation zum Rollator anhand von Distanzmessungen verschiedener Körperbereiche zum Rollator. Anhand dieser Werte wird die Haltung beurteilt. Wird ein Haltungsfehler erkannt, gibt das System eine intuitiv verständliche, dezente Rückmeldung und hilft bei der Korrektur. Als Industriepartner ist TOPRO schwerpunktmäßig für die mechanische Integration der Messelektronik am Rollator verantwortlich. Dafür nutzt das Unternehmen seine Expertise in den Bereichen Konstruktion, Teststandards und Produktion von Rollatoren. Ziel ist es, eine Lösung zu finden, mit der die Messelektronik an unterschiedlichen TOPRO-Rollatormodellen sicher befestigt und nachgerüstet werden kann. Das Nachrüsten soll dabei idealerwei-

\section{MS-Schwestern des Jahres 2016 ausgezeichnet} siums im März wurden zehn Frauen zur MSSchwester des Jahres 2016 gekürt. Mit der Auszeichnung werden zum neunten Mal in Folge das Engagement und die fachliche Expertise der MS-Schwestern gewürdigt. Sie nehmen bei der Therapie der MS eine Schlüsselrolle für ihre Patienten ein: Sie sind wichtige Ansprechpartner und unterstützen die Patienten zusätzlich neben dem Arzt.
— Im Rahmen des Interaktiven MS-Sympo-
„Die Vielzahl der heute zur Verfügung stehenden Therapieoptionen zur Behandlung der MS macht es für Betroffene zunehmend schwieriger, sich zu orientieren. Die MSSchwestern stehen den Patienten ab Therapiebeginn zur Seite, schaffen Vertrauen und bieten eine emotionale Stütze. Sie leisten damit einen wertvollen Beitrag zur Therapietreue - ergänzend zur ärztlichen Aufklärung", betonte der Schirmherr des Wettbe-

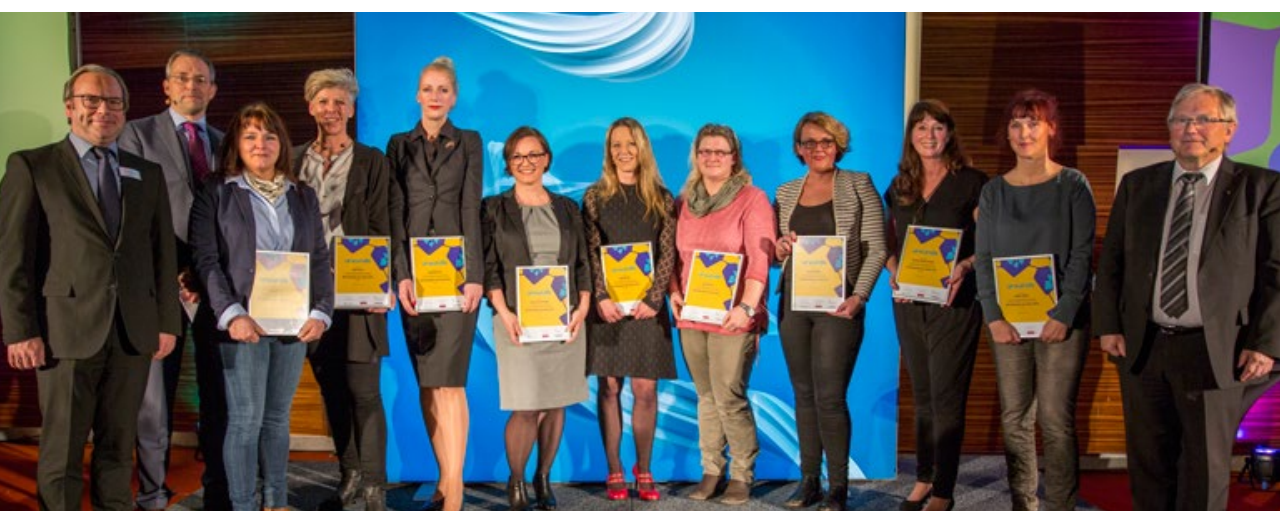

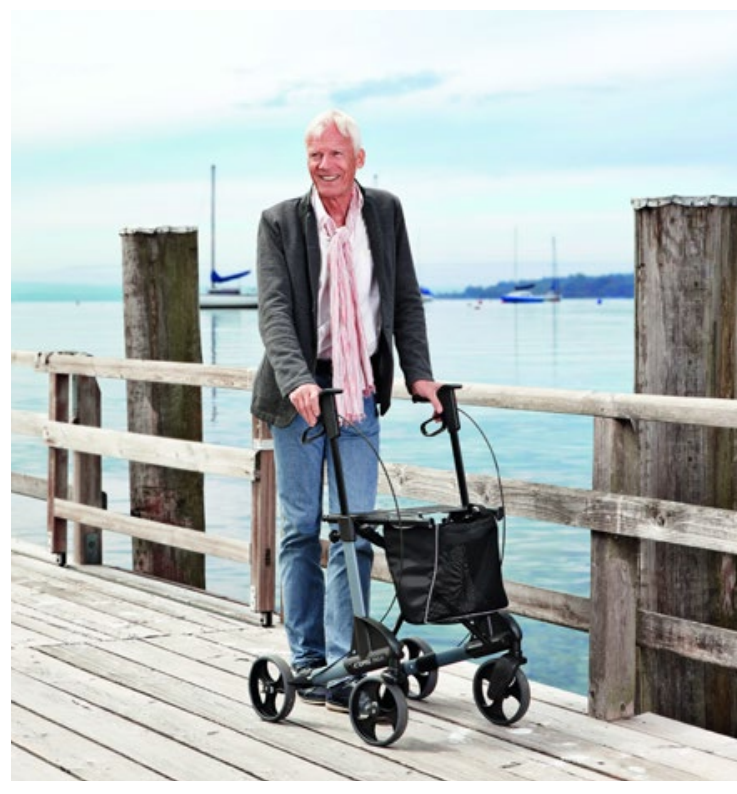

se so einfach gemacht werden, dass es auch vom Fachhändler oder von Angehörigen bzw. dem Nutzer selbst durchgeführt werden kann. www.topro.de

werbs, Prof. Dr. med. Jürgen Faiss, Chefarzt Kliniken für Neurologie Lübben und Teupitz der Asklepios Fachkliniken Brandenburg $\mathrm{GmbH}$.

In einem Leben mit Multipler Sklerose ist ein normaler Alltag manchmal schwer möglich. Hier engagiert sich Merck unter dem Motto „Endlich wieder Freiheit spüren. Endlich wieder ich." für mehr Lebensqualität und Selbstbestimmung der Patienten. Viele MS-Betroffene empfinden gerade die häufige regelmäßige Medikamenteneinnahme und die aufwendigen Kontrollen als belastend. MS-Schwestern stehen den Patienten ab Therapiebeginn zur Seite und motivieren sie, die Behandlung fortzusetzen.

Zur "MS-Schwester des Jahres 2016" wurden gekürt: Anja Arns, Yvonne Döhr, Silke Freier, Michaela Graf, Nadja von der Heyde, Anja Kanzler, Nicole Krause, Huanita Schreier-Simons und Angela Walter.

Der Wettbewerb wurde im Jahr 2008 von Merck in Kooperation mit der Zeitschrift HEILBERUFE des Springer Medizin Verlags ins Leben gerufen.

www.merckgroup.com/ www.leben-mit-ms.de 